\title{
Is there any association between absolute lymphocyte count and increased tumor-infiltrating lymphocytes in metastatic sites in advanced breast cancer patients who get benefit from eribulin treatment?
}

\author{
Kadri Altundag ${ }^{1}$
}

Received: 8 April 2020 / Accepted: 17 April 2020 / Published online: 27 April 2020

(c) Springer Science+Business Media, LLC, part of Springer Nature 2020

To the editor,

I wish to congratulate Watanabe and their colleagues for their article [1] in which they retrospectively investigated the neutrophil-to-lymphocyte ratio and absolute lymphocyte count (ALC) in HER2-metastatic breast cancer (MBC) patients receiving eribulin and assessed the utility of eribulin re-administration for further OS improvement. They reported that ALC was identified as a predictive marker for eribulin therapy, and the readministration of eribulin is considered a valid therapeutic option for further improvement of the OS in HER2-ER + MBC patients. Receptor conversion for ER, PR, and HER2 occurs frequently in the MBC. Currently, reassessing receptor status in metastases is strongly encouraged [2]. In the current study, the authors did not mention re-biopsy rates from metastatic sites among these cases and infiltrating lymphocyte distribution around the metastatic tumors. Denkert et al. reported that increased tumor-infiltrating lymphocytes were an adverse prognostic factor for survival in luminal-HER2-negative breast cancer in a pooled analysis of 3771 patients treated with neoadjuvant therapy [3]. However, there have been no data showing any association between increased TIL in metastatic site and response to eribulin or any other chemotherapeutics in MBC in the current literature. In conclusion, one would expect that there would be positive correlation between ALC and increased TIL in MBC patients who get benefit from eribulin treatment. This issue merits further investigation.

Kadri Altundag

altundag66@yahoo.com

1 MKA Breast Cancer Clinic, Tepe Prime, Cankaya, 06800 Ankara, Turkey
Funding No funding.

\section{Compliance with ethical standards}

Conflict of interest I declare that I have no conflict of interest.

Ethical approval Not applicable.

Informed consent Not applicable.

Research involving human participants or animal studies This article does not contain any studies with human participants or animals performed by any of the authors.

\section{References}

1. Watanabe J, Saito M, Horimoto Y et al (2020) A maintained absolute lymphocyte count predicts the overall survival benefit from eribulin therapy, including eribulin re-administration, in HER2negative advanced breast cancer patients: a single-institutional experience. Breast Cancer Res Treat. https://doi.org/10.1007/ s10549-020-05626-1

2. Schrijver WAME, Suijkerbuijk KPM, van Gils CH et al (2018) Receptor conversion in distant breast cancer metastases: a systematic review and meta-analysis. J Natl Cancer Inst 110(6):568-580

3. Denkert C, von Minckwitz G, Darb-Esfahani S (2018) Tumourinfiltrating lymphocytes and prognosis in different subtypes of breast cancer: a pooled analysis of 3771 patients treated with neoadjuvant therapy. Lancet Oncol 19(1):40-50

Publisher's Note Springer Nature remains neutral with regard to jurisdictional claims in published maps and institutional affiliations. 